\title{
Strategy to improve the burden of gestational diabetes in African Women: Rwandan Perspective
}

\author{
Herbert T. Mapira ${ }^{1 *}$, David K. Tumusiime ${ }^{1}$, Kevin Yarasheski², Nadine Rujeni' ${ }^{1}$, Todd W. Cade ${ }^{5}$, Eugene Mutimura ${ }^{3}$ \\ ${ }^{1}$ University of Rwanda, College of Medicine and Health Sciences, Kigali, Rwanda \\ ${ }^{2}$ Washington University School of Medicine St Louis, MO, USA \\ ${ }^{3}$ University of Rwanda \& Regional Alliance for Sustainable Development (RASD Rwanda), Kigali, Rwanda
}

\begin{abstract}
The global prevalence of gestational diabetes (GDM) in pregnant 20-49 year old women has increased to 16.9\%, although data from Africa on GDM are scarce, and risk exposure to pregnant women is unknown. Among the 288 pregnant women screened in Rwanda, $8.3 \%$ of women with FPG $>126$ $\mathrm{mg} / \mathrm{dL}$ (>6.9 mmol/L) had GDM. Age $>41$ years $(45.8 \%$ vs. $18.9 \% ; p<0.001)$, first-family history of T2D $(29.2 \%$ vs. $3.4 \% ; p<0.001)$ and gravidity $\geq 3$ pregnancies (79.2\% vs. $29.2 \%$; $p=0.05$ ) were associated with GDM. Gestational diabetes is a high risk factor for T2D, and increases the risk to T2D for women and their offspring later in life. Thus, improving maternal and child cardiometabolic health in Africa is needed through integrated gestational diabetes screening, and cost-effective sustainable strategies to build healthcare staff capacity, and to prioritize relevant evidence-based research and healthcare.
\end{abstract}

Key words: Gestational diabetes, Rwanda, Africa, Women

\section{Introduction}

The global prevalence of hyperglycemia in pregnant women $20-49$ years is increasing to $16.9 \%$ affecting over 21.4 million live births, and $\sim 90 \%$ of cases are in low- and middleincome countries (LMICs) of Africa (Mwanri et al. 2015). As Africa struggles to combat infectious diseases particularly HIV infection, tuberculosis and malaria, the challenge is to cope with increasing burden of non-communicable diseases (NCDs). The $2014 \mathrm{WHO}$ global report showed that NCDs cause most deaths worldwide, with $>80 \%$ of deaths occurring in LMICs, and mortality due to NCDs exceed communicable diseases (World Health Organization (WHO) 2010). SubSaharan Africa (SSA) bears an increasing burden of NCDs due to broader epidemiological transition from infectious to non-infection diseases as rapid urbanization and obesity increase, besides lifestyle risk determinants. The projected disease burden due to NCDs will be the greatest cause of mortality by 2035 , and prevention of NCDs would avert estimated deaths of 40 million people (World Health Organization (WHO) 2010) (Guariguata et al. 2014). Global estimate of adult 20-79 years type 2 diabetes (T2D) confirms an increase of $35.5 \%$ from 2013 to 2035, and the highest number of people with T2D will live in SSA (Guariguata et al. 2014).

Women with gestational diabetes mellitus (GDM) in SSA are among the world's poorest with highest risk to T2D, and who live on less than USD $\$ 1.45$ per day that would hardly meet overall domestic and medical demands of living for life with a chronic illness, thus competing for meager resources with infectious diseases and other emerging NCDs-e.g. cardiovascular disease (Mwanri et al. 2015). Gestational diabetes remains a major cause of maternal and prenatal morbidity in Rwandan health facilities, deserves attention due to its effect on maternal and fetus health. Other causes of maternal include post-partum hemorrhage (24\%), obstructed labor (14\%), malaria (11\%), obstetrical infections (9\%) and eclampsia (8\%), while the other causes for prenatal morbidity include neonatal illness $(51 \%)$ which comprises of GDM, prematurity (21\%), pneumopathies
(4\%), malaria, congenital anomalies and acute respiratory infections (3\%) each. (Ministry of Health 2013). Rwanda like other countries in SSA is confronted with higher morbidity and mortality rates of T2D and women with GDM are among the highest risk population to T2D (Guariguata et al. 2014). There is paucity of data from Africa on gestational diabetes and risk exposure to pregnant women is poorly known to guide policy on GDM-related risks of prenatal mortality and risk to developing T2D later in life.

\section{Methodology}

A cross-sectional study was carried out at Kimironko, Kicukiro and Muhima Health Centres located in Kigali city on 288 pregnant women. These three centers are the most urbanized among the five provinces of Rwanda. Samples were collected using a non-probability convenient sampling strategy. Two hundred and eighty eight (288) pregnant women between 24 and 28 weeks of gestation from the health centers who had not developed any type of diabetes before pregnancy and had fasted between 8 to 12 hours before glucose analysis were selected to participate in the study. Presence or absence of pre-existing diabetes mellitus was determined through testing on first antenatal visit and history of diabetes. The gestation period of pregnant women was recognized through records of prenatal care service in order to ascertain their trimester. Data was collected using laboratory diagnostic tests (Glucose Oxidase method), questionnaire and data capture sheet.

\section{Results}

Two hundred and eighyt eight (288) pregnant women of between 24 and 48 weeks of gestation from Kimironko, Kicukiro and Muhima Health Centers in Rwanda were screened for GDM. Table shows that none of the women screened were hypoglycemic with fasting plasma glucose (FPG) less than $76 \mathrm{mg} / \mathrm{dL}(<4.2 \mathrm{mmol} / \mathrm{L})$, whereas $8.3 \%$ of women with $\mathrm{FPG}>126 \mathrm{mg} / \mathrm{dL}(>6.9 \mathrm{mmol} / \mathrm{L})$ were diagnosed with gestational diabetes (American Diabetes Association 2011) Age greater than 41 years $(45.8 \%$ vs. $18.9 \% ; p<0.001)$, first-family history of T2D $(29.2 \%$ vs. $3.4 \% ; p<0.001)$ and gravidity $\geq 3$ pregnancies $(79.2 \%$ vs. 
$29.2 \% ; p=0.05$ ) were associated with gestational diabetes.

Table. Participants' characteristics by gestational diabetes mellitus $(n=288)$

\begin{tabular}{|c|c|c|c|}
\hline Characteristics & $\begin{array}{l}\text { Women with } \\
\text { GDM }(n=24)\end{array}$ & $\begin{array}{l}\text { Women } \\
\text { without GDM } \\
(\mathrm{n}=264)\end{array}$ & $\mathrm{p}$-value \\
\hline \multicolumn{4}{|l|}{ Age/ years $\mathrm{N}(\%)$} \\
\hline $21-30$ & $5(20.8 \%)$ & $129(48.9 \%$ & $<0.0001$ \\
\hline $31-40$ & $8(33.4 \%)$ & $85(32.2 \%)$ & \\
\hline$\geq 41$ & $11(45.8 \%)$ & $50(18.9 \%)$ & \\
\hline \multicolumn{4}{|l|}{ Level of income (RWF) } \\
\hline$<25,000$ & $4(16.7 \%)$ & $44(16.7 \%)$ & 0.06 \\
\hline $25,000-40,000$ & $5(20.8 \%)$ & $5721.6 \%)$ & \\
\hline $40,000-60,000$ & $6(25 \%)$ & $6524.6 \%)$ & \\
\hline$>60,000$ & $9(37.5 \%)$ & $98(37.1 \%)$ & \\
\hline \multicolumn{4}{|l|}{ Education status $\mathrm{N}(\%)$} \\
\hline No schooling & $4(16.7 \%)$ & $46(17.4)$ & 0.22 \\
\hline Primary school & $11(45.8 \%)$ & $119(45.1 \%)$ & \\
\hline Secondary or University & $9(37.5 \%)$ & $99(37.5 \%)$ & \\
\hline Pre-pregnancy BMI (kg/m²) & $24.4 \pm 3.9$ & $24.8 \pm 4.1$ & 0.23 \\
\hline Smoking N (\%) & $1(4.2 \%)$ & $10(3.8 \%)$ & 0.38 \\
\hline Alcohol use N (\%) & $3(12.5 \%)$ & $30(11.4 \%)$ & 0.18 \\
\hline \multicolumn{4}{|l|}{ Habitual Exercise } \\
\hline None & $4(16.7 \%)$ & $43(16.3 \%)$ & 0.89 \\
\hline$\geq 2$ times/week & $7(29.2 \%)$ & $77(29.2 \%)$ & \\
\hline Other (subsistence farmers) & $13(54.1 \%)$ & $144(54.5 \%)$ & \\
\hline Gravidity $N(\%) \geq 3$ pregnancies & $19(79.2 \%)$ & $77(29.2 \%)$ & 0.05 \\
\hline Family history of T2D N (\%) & $7(29.2 \%)$ & $9(3.4 \%)$ & $<0.001$ \\
\hline Family history of HTN N (\%) & $3(12.5 \%)$ & $34(12.9 \%)$ & 0.84 \\
\hline \multicolumn{4}{|l|}{ Blood pressure values (mmHg) } \\
\hline Systolic BP (mmHg) & $124.6 \pm 17.7$ & $124.5 \pm 18.0$ & 0.57 \\
\hline Diastolic BP (mmHg) & $84.0 \pm 12.1$ & $84.5 \pm 11.0$ & \\
\hline \multicolumn{4}{|l|}{ Other infections N (\%) } \\
\hline HIV infection & $1(8.3)$ & $11(91.7)$ & 0.67 \\
\hline HCV/HBV co-infection & - & - & - \\
\hline
\end{tabular}

BMI, body mass index; T2D, type 2 diabetes mellitus; HTN, hypertension

\section{Discussion}

Our finding of $8.3 \%$ GDM among Rwandan women is largely comparable with data from developed countries: $3.2-7.6 \%$ in the US and $2.0-11.6 \%$ in Europe,(Schneider et al. 2012) as well as data from Africa: $4.5 \%-13.4 \%$ in Nigeria, (Anzaku \& Musa 2013) $3.7 \%$ in Ethiopia (Seyoum et al. 1999) and $3.8-8.8 \%$ in South Africa .(Mamabolo et al. 2007). Although the prevalence of GDM highly depends on method and criteria used, pre-pregnancy $\mathrm{BMI}$, a strong predictor of GDM in other studies,(Schneider et al. 2012; Mamabolo et al. 2007) was not associated with GDM- perhaps due to differences in dietary behavioral factors and balance between nutrient metabolism and degree of adipogenesis, as well as innate genetic predisposition. Age and firstfamily history of diabetes were associated with GDM as consistently reported elsewhere (Aberg et al. 2002).

Gestational diabetes, a major risk factor for T2D is slowly and strongly creeping into Rwandan health care systems like in other health facilities in SSA. About $~ 7 \%$ of all pregnancies in Africa are complicated by gestational diabetes, and up to $14 \%$ of all pregnancies develop gestational diabetesrelated complications (Mwanri et al. 2015). Although GDM is a major cause of prenatal and maternal morbidity in Rwanda, it is still on the rise such that by the time it peaks its effects will be confounded. Early screening and management of GDM in African women is imperative due to women's increased risk to T2D, with a conversion rate of $3 \%$ per year,(Guariguata et al. 2014) and $~ 50 \%$ of women may develop non-insulin dependent T2D within the next 5-7 years (Mwanri et al. 2015). The associated maternal hyperglycemia causes the fetus to secrete more insulin, resulting in stimulation of fetal growth and macrosomia, and their offspring are at an increased risk of developing T2D later in life.(Mwanri et al. 2015; Guariguata et al. 2014) As some African countries acclaim remarkable progress to improve maternal and child health-millennium development goal five, much is still needed to improve maternal and child cardiometabolic health, that affects their offspring wellbeing through intrauterine mechanisms (Gaillard et al. 2014). Rwanda's healthcare system with public health centers and poste des santés at the base of healthcare pyramid deep, supports the potential to provide integrated screening for gestational diabetes, but this will need strong cost-effective and sustainable policies and strategies that focus on integrated approach of building healthcare staff capacity and prioritize homegrown socially and culturally relevant evidence-based research and healthcare.

\section{Conclusion}

In conclusion the findings of this study show that the prevalence of GDM was $8.3 \%$ and the most affected age was over 41 years. Family history of diabetes mellitus, and gravidity $\geq 3$ pregnancies were risk factors for developing GDM.

Other factors like family history of hypertension, levels of income and education, BMI, smoking, alcohol use 
exercise and other infections were not significant factors for developing GDM.

\section{Limitations of the Study and future Directions}

These findings are limited by small sample size, lack of inclusion of women from rural facilities and inability to assess associations of GDM with birth weight as participants delivered at different sites. Nevertheless, the University of Rwanda is poised to implement nation-wide screening of pregnant women between 24 to 48 weeks of gestation at a greater risk for T2D to curtail potential effects of T2D. We will continue to foster partnerships with national healthcare facilities and international collaborators to enhance integrated optimal models of T2D care and focus on basic, clinical and translation research.

Our research and community engagement strategy prioritize the need for strong scientific evidence on T2D that poses the greatest burden. We will continue to coordinate efforts and reinforce use of limited resources -integrating laboratory, clinical investigations and research shared core facilities. We are striving to develop an integrated infrastructure to utilize more gold-standard techniques of hyperinsulinemiceuglycemic clamp and oral glucose tolerance test to assess diabetes, as well as imaging, histology and cytology for diagnosis of relevant NCDs. Our vision is to develop more high-level research and clinical facilities such as mass spectrometry, genetic molecular laboratory equipment for DNA sequencing and genotyping as well as computerized tomography as integrated and shared core facilities. Besides, we are working to develop specimen bank facilities at referral and teaching laboratory facilities as well as large datasets that will be shared among centers, for teaching, healthcare and research, additionally supporting provincial institutions to deliver more state-of-art clinical care on a wide range of non-communicable diseases.

\section{Acknowledgement and funding}

We are grateful to funding received from the University of Rwanda, Regional Alliance for Sustainable Development, Rwanda, and Washington University of Medicine St Louis MO, USA.

\section{Conflict of interests}

None

\section{Authors' contributions}

$\mathrm{H}$ M played a role in the conception, design, interpretation, and writing of the manuscript; D K T contributed with the conception and design of the manuscript; $\mathrm{K} Y$ contributed with Data analysis; NR contributed with conception and design of the manuscript; TW C contributed with Data analysis; E M contributed with the conception, and writing of the manuscript.

\section{References}

Aberg, A.E.B. et al., 2002. Predictive factors of developing diabetes mellitus in women with gestational diabetes. Acta obstetricia et gynecologica Scandinavica, 81(1), pp.11-6. Available at: http://www.ncbi.nlm.nih. gov/pubmed/11942881.

American Diabetes Association, 2011. Diagnosis and classification of diabetes mellitus. Diabetes care, 34 Suppl 1, pp.S62-9. Available at: http://www.pubmedcentral.nih.gov/articlerender.fcgi? artid=3006051\&tool = pmcentrez\&rendertype = abstract \nhttp: //www.ncbi. nlm.nih.gov/pubmed/9118500.

Anzaku, A.S. \& Musa, J., 2013. Prevalence and associated risk factors for gestational diabetes in Jos, North-central, Nigeria. Archives of gynecology and obstetrics, 287(5), pp.859-63. Available at: http:// www.ncbi.nlm.nih.gov/pubmed/23224650.

Gaillard, R. et al., 2014. Childhood cardiometabolic outcomes of maternal obesity during pregnancy: The generation r study. Hypertension, 63(4), pp.683-691.

Guariguata, L. et al., 2014. Global estimates of diabetes prevalence for 2013 and projections for 2035. Diabetes Research and Clinical Practice, 103(2), pp.137-149. Available at: http://dx.doi.org/10.1016/j. diabres.2013.11.002.

Mamabolo, R.L. et al., 2007. Prevalence of gestational diabetes mellitus and the effect of weight on measures of insulin secretion and insulin resistance in third-trimester pregnant rural women residing in the Central Region of Limpopo Province, South Africa. Diabetic medicine : a journal of the British Diabetic Association, 24(3), pp.233-9. Available at: http://www.ncbi. nlm.nih.gov/pubmed/17263763 [Accessed January 7, 2016].

Ministry of Health, 2013. Rwanda Annual Health Statistics Booklet, Kigali. Available at: http://www.moh.gov. rw/fileadmin/templates/HMIS_Docs/Rwanda_Annual_Health_Statistics_Booklet_2013_signed.pdf.

Mwanri, A.W. et al., 2015. Gestational Diabetes Mellitus in sub-Saharan Africa: Systematic Review and Meta-Regression on Prevalence and Risk Factors. Tropical Medicine \& International Health, p.n/a-n/a. Available at: http://dx.doi.org/10.1111/tmi.12521.

Schneider, S. et al., 2012. The prevalence of gestational diabetes in advanced economies. Journal of perinatal medicine, 40, pp.1-10. Available at: http://www. ncbi.nlm.nih.gov/pubmed/22706228.

Seyoum, B. et al., 1999. Prevalence of gestational diabetes mellitus in rural pregnant mothers in northern Ethiopia. Diabetes Research and Clinical Practice, 46, pp.247-251.

World Health Organization (WHO), 2010. Global Status Report on Non-Communicable Diseases. Global Status Report on Non-Communicable Diseases. Available at: http://www.who.int/nmh/publications/ ncd_report_full_en.pdf. 